PROCEEDINGS OF THE

AMERICAN MATHEMATICAL SOCIETY

Volume 130, Number 1, Pages 39-47

S 0002-9939(01)06373-0

Article electronically published on August 7, 2001

\title{
UNIFORM SUBELLIPTIC ESTIMATES ON SCALED CONVEX DOMAINS OF FINITE TYPE
}

\author{
JEFFERY D. MCNEAL
}

(Communicated by Steven R. Bell)

\begin{abstract}
We show that a uniform subelliptic estimate for the $\bar{\partial}$-Neumann problem holds on a certain family of convex domains of finite type.
\end{abstract}

Let $\Omega$ be a smoothly bounded convex domain and let $p \in b \Omega$ be a point of finite type. In this note we show that a uniform subelliptic estimate for the $\bar{\partial}$-Neumann problem on $(0,1)$-forms holds on the family of rescaled domains associated to $\Omega$ as defined in Mc1]. Uniformity here means that the constituents of the subelliptic estimate (the constant on the right hand side, the Sobolev index, the size of the neighborhood) can be chosen so that the estimate holds, with these constituents, on every domain in the rescaled family.

This result was earlier stated in [Mc1] as Proposition 4.1. While the argument given there is substantially correct, the proof of Proposition 4.1 used a plurisubharmonic constructed in Proposition 3.1 of [Mc1] and there is a gap in the proof of Proposition 3.1. This error was kindly pointed out to the author by Yves Dupain. The essential function in that proposition, $\phi$, is correctly constructed; the gap occurs when the support of $\phi$ is cut-down to the polydisc $P_{\epsilon}(q)$ by using the isotropic support functions borrowed from [FoSi]. As we will indicate below, one way to repair the proof of Proposition 3.1 is to substitute the recently obtained non-isotropic support functions in $\mathrm{DiF}$. for the isotropic support functions; the remainder of the proof of Proposition 3.1 is then as given in [Mc1].

However, the compactly supported plurisubharmonic functions of Proposition 3.1 in [Mc1] are not necessary for demonstrating that a uniform subelliptic estimate holds on the rescaled domains nor are they required for the proof of the main results in [Mc1], i.e. the estimates on the Bergman kernel, associated to $\Omega$, and its derivatives. Once a uniform subelliptic is known to hold, all the results in [Mc1] are valid as one may simply replace the functions of Proposition 3.1 by the function $\phi$ mentioned above in all proofs.

Because of the confusion our error has caused and because several authors have used our Bergman kernel estimates for other applications, e.g. Cum1], Cum2, KrLi], McSt], it seems important to clarify the situation and directly establish the foundational inequality. We take this opportunity to give two separate arguments which show that a uniform subelliptic estimate holds on the rescaled domains.

Received by the editors February 3, 2000.

1991 Mathematics Subject Classification. Primary 32W05.

This research was supported by an Alfred P. Sloan fellowship and by a grant from the National Science Foundation.

(C)2001 American Mathematical Society 


\section{Preliminaries}

We fix notation and recall some results from [Mc1]. Throughout, $\Omega \subset \subset \mathbb{C}^{n}$ is a smoothly bounded, convex domain of finite type $M$ defined by a real-valued function $r$ with convex infralevel sets. We recall the definition of finite type. If $g$ is a sufficiently smooth function defined in a neighborhood of a point $p \in \mathbb{C}^{n}$, we denote the order of $g$ at $p$ by $\nu_{p}(g)$; usually $p$ will be understood in context and we will drop the subscript. For a mapping $G: \mathbb{C}^{n} \rightarrow \mathbb{C}^{m}$ defined near $p, \nu(G)$ will denote the minimum order of vanishing of the components of $G$. The D'Angelo 1-type of $p \in b \Omega$ is defined as

$$
\Delta_{1}(p)=\sup \left\{\frac{\nu_{p}(r \circ \gamma)}{\nu_{0}(\gamma)}: \gamma: \mathbb{C} \rightarrow \mathbb{C}^{n}, \gamma(0)=p, \gamma \text { complex analytic }\right\} .
$$

The notion of finite type simplifies in the convex case. Let $L$ be an arbitrary complex line through the origin in $\mathbb{C}^{n}$. If $\lambda$ is a vector such that $s \rightarrow s \lambda$ parameterizes $L$, we define the order of contact of $L$ with $b \Omega$ at $p$ to be the least positive integer $M$ such that for some $a, b$ with $a+b=M$,

$$
\left|D_{s}^{a} D_{\bar{s}}^{b} r(p+s \lambda)\right|_{s=0} \neq 0 .
$$

Denote this order of contact by $\nu_{p}(r \circ L)$. If we normalize the length of the vectors by, say, requiring that $|\lambda|=1$, the definition is independent of the choice of $\lambda$. In Mc2, it is shown that $\Delta_{1}(p)=\sup _{L} \nu_{p}(r \circ L)$ on convex domains.

Let $p \in b \Omega$ be temporarily fixed and let $U$ be a small neighborhood of $p$. If $q \in U \cap \bar{\Omega}$ and $\epsilon>0$, the coordinate construction in Mc1] gives coordinates $\left(z_{1}^{q, \epsilon}, \ldots, z_{n}^{q, \epsilon}\right)$ centered at $q$, which are an affine transformation of the standard coordinates on $\mathbb{C}^{n}$, in which all the computations simplify. Call these coordinates the $(q, \epsilon)$-extremal coordinates. Associated to these coordinates are positive real numbers $\tau_{1}(q, \epsilon), \ldots, \tau_{n}(q, \epsilon)$ defined as follows. Let $\tau_{1}(q, \epsilon)=\epsilon$. Write $\left(z_{1}, \ldots, z_{n}\right)=\left(z_{1}^{q, \epsilon}, \ldots, z_{n}^{q, \epsilon}\right)$ and set, for each $2 \leq l \leq M$ and $2 \leq j \leq n$,

$$
A_{l}^{j}(q)=\max \left\{\left|\frac{\partial^{l}}{\partial z_{j}^{a} \partial \bar{z}_{j}^{b}} r(q)\right|: a+b=l\right\} \text {. }
$$

Then for $2 \leq j \leq n$,

$$
\tau_{j}(q, \epsilon)=\min \left\{\left(\epsilon / A_{l}^{j}(q)\right)^{1 / l}: 2 \leq l \leq M\right\} .
$$

The polydisc determined by the numbers $\tau_{j}(q, \epsilon)$ is denoted

$$
P_{\epsilon}(q)=\left\{z:\left|z_{i}^{q, \epsilon}\right|<\tau_{i}(q, \epsilon), \quad \forall i\right\} .
$$

If $\lambda$ is a vector in $\mathbb{C}^{n}$, we set

$$
\sigma(q, \lambda, \epsilon)=\sup \{D: r(q+s \lambda)-r(q) \leq \epsilon,|s| \leq D\}
$$

The main properties of the quantities above are summarized in the following proposition. The proofs are contained in [Mc1] (see also McSt for elaboration). Here, and in the remainder of the note, we use the notation $A \lesssim B, A \gtrsim B$, and $A \approx B$ in the same way as [Mc1].

Proposition 1. (i) If $u_{1}, \ldots, u_{n}$ are the unit vectors determined by the axes $z_{1}^{q, \epsilon}, \ldots, z_{n}^{q, \epsilon}$ respectively, and $\lambda=\sum a_{i} u_{i}, a_{i} \in \mathbb{C}$, then

$$
\sigma(q, \lambda, \epsilon) \approx\left(\sum_{i=1}^{n} \frac{\left|a_{i}\right|}{\tau_{i}(q, \epsilon)}\right)^{-1}
$$


(ii) There exists a constant $C>0$, independent of $q^{1}, q^{2} \in U \cap \bar{\Omega}$ and $\epsilon$, so that if $P_{\epsilon}\left(q^{1}\right) \cap P_{\epsilon}\left(q^{2}\right) \neq \emptyset$,

$$
P_{\epsilon}\left(q^{1}\right) \subset C P_{\epsilon}\left(q^{2}\right) .
$$

(iii) For any $d>0$, there exists a constant $C(d)$, independent of $q$ and $\epsilon$, such that

$$
P_{d \epsilon}(q) \subset C(d) P_{\epsilon}(q) .
$$

(iv) If $q^{2} \in P_{\epsilon}\left(q^{1}\right)$ and $\lambda$ is arbitrary,

$$
\sigma\left(q^{1}, \lambda, \epsilon\right) \approx \sigma\left(q^{2}, \lambda, \epsilon\right) .
$$

(v) For $q^{1}, q^{2} \in U \cap \bar{\Omega}$, define

$$
\mathcal{M}\left(q^{1}, q^{2}\right)=\inf \left\{\epsilon: q^{2} \in P_{\epsilon}\left(q^{1}\right)\right\} .
$$

Then $\mathcal{M}$ is a pseudometric, i.e.

$$
\begin{aligned}
& \mathcal{M}\left(q^{1}, q^{2}\right) \approx \mathcal{M}\left(q^{2}, q^{1}\right), \\
& \mathcal{M}\left(q^{1}, q^{2}\right) \lesssim \mathcal{M}\left(q^{1}, q^{3}\right)+\mathcal{M}\left(q^{3}, q^{2}\right) .
\end{aligned}
$$

(vi) For all $j$, if $1>c>0$,

$$
c^{1 / 2} \tau_{j}(q, \epsilon) \leq \tau_{j}(q, c \epsilon) \leq c^{1 / M} \tau_{j}(q, \epsilon) .
$$

The domain $\Omega$ is rescaled using the coordinates $z^{q, \epsilon}$ and the weights $\tau(q, \epsilon)$. Let $q$ and $\epsilon$ be temporarily fixed (for simplicity write $\left(z_{1}, \ldots, z_{n}\right)$ for the $(q, \epsilon)$-extremal coordinates), and define the mapping

$$
\left(z_{1}, \ldots, z_{n}\right)=\Phi^{q, \epsilon}\left(w_{1}, \ldots, w_{n}\right)=\left(\tau_{1}(q, \epsilon) w_{1}, \ldots, \tau_{n}(q, \epsilon) w_{n}\right) .
$$

Note that $\Phi^{q, \epsilon}(0)=0=\left(z_{1}(q), \ldots, z_{n}(q)\right)$. If $V$ is a neighborhood of 0 , we define

$$
\Omega^{q, \epsilon} \cap V=\left\{w \in V: \rho_{\epsilon}(w)<0\right\},
$$

where

$$
\rho_{\epsilon}(w)=\frac{1}{\epsilon} r \circ \Phi^{q, \epsilon}(w) .
$$

Finally, we recall the estimate referred to in the title. If $U$ is a neighborhood of $p \in b \Omega$, let $\mathcal{D}^{0,1}(U)$ denote the smooth $(0,1)$-forms, supported in $U$, which satisfy

$$
\sum_{j=1}^{n} \frac{\partial r}{\partial z_{j}} u_{j}=0 \quad \text { on } b \Omega,
$$

where $u=\sum u_{j} d \bar{z}_{j}$. A subelliptic estimate, of order $\kappa$, for the $\bar{\partial}$-Neumann problem on $(0,1)$-forms holds in $U$ if there exists a constant $C$ such that

$$
\|\| u \|\left.\right|_{\kappa} ^{2} \leq C\left(\|\bar{\partial} u\|^{2}+\|\vartheta u\|^{2}+\|u\|^{2}\right), \quad u \in \mathcal{D}^{0,1}(U) .
$$

Here $\|\cdot\|$ denotes the Euclidean $L^{2}$ norm and $\||\cdot|\|_{\kappa}$ denotes the tangential Sobolev norm of order $\kappa$. 


\section{UNIFORMITY OF TYPE UNDER RESCALING}

First, we introduce a measurement related to (3). Let $\lambda \in \mathbb{C}^{n}$ be a vector and consider the parameterized complex line $s \rightarrow s \lambda, s \in \mathbb{C}$. Let $D_{s}^{a}=\partial^{a} / \partial s^{a}$, $D_{\bar{s}}^{b}=\partial^{b} / \partial \bar{s}^{b}$, and set

$$
c_{a b}^{\lambda}(q)=\left.D_{s}^{a} D_{\bar{s}}^{b} r(q+s \lambda)\right|_{s=0} .
$$

Also define, for $1 \leq l \leq M$,

$$
A(q, l, \lambda)=\max \left\{\left|c_{a b}^{\lambda}(q)\right|: a+b=l\right\},
$$

and, for $\epsilon>0$,

$$
\theta(q, \lambda, \epsilon)=\min \left\{\left(\frac{\epsilon}{A(q, l, \lambda)}\right)^{1 / l}: 1 \leq l \leq M\right\} .
$$

We shall show that $\theta(q, \lambda, \epsilon)$ is comparable to $\sigma(q, \lambda, \epsilon)$ on a convex domain of finite type. First, we recall the following result from [B-N-W]; see also [B-C-D].

Lemma 2. Let $N \in \mathbb{N}$. There exists a constant $C=C(N)$ such that

(a) If $P$ is a polynomial of degree $\leq N$ which is convex on $[-1,1], P(x)=$ $\sum_{j=1}^{N} c_{j} x^{j}$, then

$$
\max \{|P(-x)|,|P(x)|\} \geq C \sum_{j=1}^{N}\left|c_{j}\right||x|^{j}, \quad-1 \leq x \leq 1 .
$$

(b) If $P$ is a polynomial on $\mathbb{C}$ of degree $\leq N$ which is convex on $|z| \leq 1, P(z)=$ $\sum_{1 \leq a+b \leq d}^{N} c_{a b} z^{a} \bar{z}^{b}$, then

$$
\sup _{|z|=h}|P(z)| \geq C \sum_{1 \leq a+b \leq d}^{N}\left|c_{a b}\right| h^{a+b}, \quad 0 \leq h \leq 1 .
$$

Proof. The result follows from Lemma 2.1 in B-N-W]. However, the result there is not written exactly as above and we mention the small modifications which give the stated version.

For $(\mathrm{a})$, let $\mathcal{P}_{x}^{N}=\left\{P(x)=\sum_{j=1}^{N} c_{j} x^{j}\right\}$ be the set of all polynomials, of $x \in \mathbb{R}$, of degree $\leq N$ which vanish at 0 . Let $C \mathcal{P}_{x}^{N}$ be the subset of $\mathcal{P}^{N}$ consisting of elements which are convex on $[-1,1]$. Consider the following two functionals:

$$
\begin{aligned}
& \|P\|_{1}=\max \{|P(1)|,|P(-1)|\}, \\
& \|P\|_{2}=\sum_{j=1}^{N}\left|c_{j}\right| .
\end{aligned}
$$

$\|\cdot\|_{2}$ is obviously a norm on $\mathcal{P}_{x}^{N}$. However, $\|\cdot\|_{1}$ is a norm on $C \mathcal{P}^{N}$, since $\|P\|_{1}=0$ implies that $P(1)=P(-1)=P(0)=0$, and convexity then forces $P \equiv 0$. Since $\mathcal{P}_{x}^{N}$ is finite dimensional, there exists a constant $C>0$ such that

$$
\|P\|_{1} \geq C\|P\|_{2}, \quad P \in C \mathcal{P}_{x}^{N} \text {. }
$$

If $x \in[-1,1]$, apply (10) to the polynomial $Q(t)=P(t|x|)$ to obtain (a).

The proof of (b) is basically the same. Let $\mathcal{P}_{z}^{N}=\left\{P(z)=\sum_{1 \leq a+b \leq N} c_{a b} z^{a} \bar{z}^{b}\right\}$ be the set of all polynomials, of $z \in \mathbb{C}$, of degree $\leq N$ which vanish at 0 . Let $C \mathcal{P}_{z}^{N}$ be the subset of $\mathcal{P}_{z}^{N}$ consisting of elements which are convex on $|z| \leq 1$. The 
relevant functionals are: $\|P\|_{3}=\sup \{|P(z)|:|z|=1\}$ and $\|P\|_{4}=\sum_{1 \leq a+b \leq d}^{N}\left|c_{a b}\right|$. As before, convexity shows that $\|\cdot\|_{3}$ is non-degenerate on $C \mathcal{P}_{z}^{N} \backslash 0$. The finite dimensionality of $\mathcal{P}_{z}^{N}$ and scaling then give (b).

Proposition 3. Let $p \in b \Omega$ and let $U$ be a neighborhood of $p$ such that $\Delta_{1}(\tilde{p}) \leq M$ for all $\tilde{p} \in U \cap b \Omega$. For all $\epsilon>0$ sufficiently small and $|\lambda|=1$, there exist constants such that

$$
\theta(q, \lambda, \epsilon) \approx \sigma(q, \lambda, \epsilon), \quad q \in U .
$$

Proof. Restrict $r$ to the line $s \rightarrow s \lambda, s \in \mathbb{C}$; for $N \geq 2$ Taylor's theorem gives

$$
r(q+s \lambda)-r(q)=\sum_{2 \leq a+b \leq N} c_{a b}^{\lambda}(q) s^{a} \bar{s}^{b}+\mathcal{O}\left(|s|^{N+1}\right) .
$$

Let $N=M$. If $|s| \leq \theta(q, \lambda, \epsilon)$, it follows from (9) and the fact that $\theta(q, \lambda, \epsilon) \lesssim \epsilon^{1 / M}$ that $|r(q+s \lambda)-r(q)| \lesssim \epsilon$. Thus, definition (5) implies $\sigma(q, \lambda, \epsilon) \gtrsim \theta(q, \lambda, \epsilon)$.

For the reverse inclusion, let $e=\min \left\{l: \theta(q, \lambda, \epsilon)=\left(\frac{\epsilon}{A(q, l, \lambda)}\right)^{1 / l}\right\}$. Letting $h=\theta(q, \lambda, \epsilon)$, Lemma 2 implies

$$
\begin{aligned}
\sup _{s=r}|r(q+s \lambda)-r(q)| & \gtrsim \sum_{1 \leq a+b \leq M}\left|c_{a b}^{\lambda}(q)\right| \theta(q, \lambda, \epsilon)^{a+b}+\mathcal{O}\left(\theta(q, \lambda, \epsilon)^{M+1}\right) \\
& \geq A(q, e, \lambda) \theta(q, \lambda, \epsilon)^{e} \\
& \geq \epsilon .
\end{aligned}
$$

Thus, $\sigma(q, \lambda, \epsilon) \lesssim \theta(q, \lambda, \epsilon)$.

We mention a simple

Corollary 4. Let $p \in U \cap b \Omega$ and suppose that there exists a constant $C$ so that for any $\lambda \in \mathbb{C}^{n},|\lambda|=1$,

$$
\sigma(p, \lambda, \delta) \leq C \delta^{1 / K}
$$

for all small $\delta>0$. Then $\Delta_{1}(p) \leq K$.

Proof. Let $L$ be an arbitrary complex line parameterized by $s \rightarrow s \lambda$. Proposition 3 and (11) imply that $\theta(p, \lambda, \delta) \lesssim \delta^{1 / K}$, for an independent constant. It follows from (9) that $A(p, K, \lambda) \gtrsim 1$, and this implies that the order of contact of $L$ with $b \Omega$ at $p$ is $\leq K$.

We now show that the type is not increased by scaling with respect to the $\tau$ structure.

Proposition 5. There exists a constant $C>0$ such that: if $q \in U \cap \Omega, \epsilon>0$, and $L$ is a complex line through $0=\Phi_{q, \epsilon}(q)$, then

$$
\left|D^{\alpha}\left(\rho_{\epsilon} \circ L\right)(0)\right| \geq C
$$

for some $\alpha,|\alpha| \leq M$. Thus $0 \in b \Omega_{q, \epsilon}$ is of finite type at most $M$, independent of $q$ and $\epsilon$.

Proof. It is convenient to extend the definitions of $\sigma(q, \lambda, \epsilon)$ and $\theta(q, \lambda, \epsilon)$ to functions other than $r$. If $f$ is defined in a neighborhood of $q \in \mathbb{C}^{n}, \lambda \in \mathbb{C}^{n}$, and $\epsilon>0$, define

$$
\sigma(f ; q, \lambda, \epsilon)=\sup \{D:|f(q+s \lambda)-f(q)| \leq \epsilon,|s| \leq D\} .
$$


If $f$ is $C^{\infty}$ near $q$ and $M \in \mathbb{N}$ is given, set

$$
A(f ; q, l, \lambda)=\max \left\{\left|D_{s}^{a} D_{\bar{s}}^{b} f(q+s \lambda)\right|_{s=0}: 1 \leq l \leq M\right\},
$$

and define

$$
\theta(f ; q, \lambda, \epsilon)=\min \left\{\left(\frac{\epsilon}{A(f ; q, l, \lambda)}\right)^{1 / l}: 1 \leq l \leq M\right\}
$$

We shall use the coordinates $\left(z_{1}^{q, \epsilon}, \ldots, z_{n}^{q, \epsilon}\right)$ associated to $q$ and $\epsilon$ to express all vectors. Let $\lambda=\left(\lambda_{1}, \ldots, \lambda_{n}\right)$ be a vector in $\mathbb{C}^{n}$ such that $s \rightarrow s \lambda$ parameterizes $L$ and let $\tilde{\lambda}=\left(\tau_{1}(q, \epsilon) \lambda_{1}, \ldots, \tau_{n}(q, \epsilon) \lambda_{n}\right)$. For $\delta>0$, it follows directly from (6) and (12) that

$$
\sigma\left(\rho_{\epsilon} ; q, \lambda, \delta\right)=\sigma(r ; q, \tilde{\lambda}, \delta \epsilon)
$$

However (i) and (vi) in Proposition 1 imply

$$
\sigma(r ; q, \tilde{\lambda}, \delta \epsilon) \approx\left(\sum_{l=1}^{n} \frac{\tau_{l}(q, \epsilon)\left|\lambda_{l}\right|}{\tau_{l}(q, \delta \epsilon)}\right)^{-1} \leq \delta^{1 / M} .
$$

The result now follows from Corollary 4.

A careful examination of the proofs of Theorems 9.1 and 9.2 in Cat show that the family of weight constructed in those theorems may be contructed (locally) on each of the domains $\Omega_{q, \epsilon}$ and, because of Proposition 5, the constants involved are independent of $q$ and $\epsilon$. The main result in Cat then implies that (7) holds, for a uniform $U, C$, and $\kappa$, on each of the domains $\Omega_{q, \epsilon}$. We point out that the value of $\kappa$ given by Catlin's construction is rather small; see the introduction in [Cat for an estimate of its size.

\section{USING NON-ISOTROPIC SUPPORT FUNCTIONS}

For $q \in b \Omega$, let $N_{q}$ denote the unit normal to $b \Omega$ at $q$. If $T_{q}$ is a unit tangent vector to $b \Omega$ at $q$ and $s \in \mathbb{C}$, let

$$
a_{k l}\left(q, T_{q}\right)=\left.\frac{\partial^{k+l}}{\partial s^{k} \partial \bar{s}^{l}} r\left(q+s T_{q}\right)\right|_{s=0}
$$

and set

$$
A_{j}\left(q, T_{q}\right)=\sum_{k+l=j}\left|a_{k l}\left(q, T_{q}\right)\right| .
$$

Let $B(q, R)$ denote the ball in $\mathbb{C}^{n}$ centered at $q$ of radius $R$.

Recently, Diederich and Fornæss proved the following

Theorem $([\overline{\mathrm{DiF}}])$. There exist constants $C, R>0$ so that: for every $q \in b \Omega$, there exists a holomorphic function, $S_{q}(z)$, on $B(q, R)$ satisfying

(i) $S_{q}(q)=0$,

(ii) if $z \in \bar{\Omega} \cap B(q, R)$ and $z=a N_{q}+b T_{q}$, then

$$
\operatorname{Re} S_{q}(z) \leq-C\left(|\operatorname{Re} a|+|\operatorname{Im} a|^{2}+\sum_{j=2}^{M} A_{j}\left(q, T_{q}\right)|b|^{j}\right) .
$$


Fix $q \in b \Omega$, let $\epsilon>0$, and let $\left(z_{1}, \ldots, z_{n}\right)$ be the $(q, \epsilon)$-extremal coordinates.

From the coordinate construction, $\operatorname{Re} z_{1} \leq 0$ in $\bar{\Omega} \cap U$, so $\log z_{1}$ is well defined locally, and

$$
\operatorname{Re}\left(i z_{1}^{1 / 2}\right) \leq-\frac{1}{2}\left|z_{1}\right|^{1 / 2}, \quad z \in \bar{\Omega} \cap U
$$

On a small neighborhood of $q$, contained both in $U$ and in $B(q, R)$, define

$$
C_{q}(z)=i z_{1}^{1 / 2}+S_{q}(z)
$$

using the $\left(z_{1}, \ldots, z_{n}\right)$ coordinates.

Proposition 6. If $z \in\left(\bar{\Omega} \backslash P_{\epsilon}(q)\right) \cap U$, then

$$
\operatorname{Re} C_{q}(z) \lesssim-\epsilon
$$

for a constant independent of $q$ and $\epsilon$.

Proof. Write $z \in\left(\bar{\Omega} \backslash P_{\epsilon}(q)\right) \cap U$ as $z=a N_{q}+b T_{q}, a, b \in \mathbb{C}$, for $T_{q}$ some tangent vector. If $|b| \geq c_{1} \sigma\left(q, T_{q}, \epsilon\right)$ for a small, independent constant $c_{1}$, then

$$
\begin{aligned}
\operatorname{Re} C_{q}(z) & \leq \operatorname{Re} S_{q}(z) \\
& \leq-C \sum_{j=2}^{M} A_{j}\left(q, T_{q}\right)|b|^{j} \\
& \leq-C c_{1} \sum_{j=2}^{M} A_{j}\left(q, T_{q}\right) \sigma\left(q, T_{q}, \epsilon\right)^{j} \\
& \lesssim-\epsilon .
\end{aligned}
$$

The last inequality follows from (9).

However, if $|b| \leq c_{1} \sigma\left(q, T_{q}, \epsilon\right)$, then $z \notin P_{\epsilon}(q)$ implies that $|a| \gtrsim \epsilon$. It follows directly from (14) that

$$
\operatorname{Re} C_{q}(z) \lesssim-\epsilon^{1 / 2}<<-\epsilon .
$$

This completes the proof.

To repair the proof of Proposition 3.1 in [Mc1, start with the function $\phi$ constructed in the early part of that proposition: $\phi$ is plurisubharmonic on $\Omega,|\phi| \leq 1$ on $\Omega \cap U$, and

$$
\sum_{i, j=1}^{n} \frac{\partial^{2} \phi}{\partial z_{i} \partial \bar{z}_{j}}(z) \xi_{i} \bar{\xi}_{j} \gtrsim \sum_{i=1}^{n} \frac{\left|\xi_{i}\right|^{2}}{\tau_{i}(q, \epsilon)^{2}}, \quad z \in P_{\epsilon}(q),
$$

where $\left(z_{1}, \ldots, z_{n}\right)$ are the $(q, \epsilon)$-extremal coordinates and the constant is independent of $q$ and $\epsilon$. Also, $A=\phi(q)$ is independent of $q$ and $\epsilon$.

Proposition 6 above shows that $\omega(z)=\frac{1}{K \epsilon} C_{q}(z)$, for a constant $K$ independent of $q$ and $\epsilon$, satisfies

(i) $\omega(q)=0$, and

(ii) $\omega(z) \leq-1$ if $z\left(\bar{\Omega} \backslash P_{\epsilon}(q)\right) \cap U$.

Let $\chi$ be a convex, increasing function on $\mathbb{R}$ with the properties $\chi(x)=0$ if $x<$ $A / 2$ and $\chi^{\prime}(x)>0$ if $x \geq A / 2$. Set $h(z)=\phi(z)+\omega(z)$ and define $F_{q, \epsilon}(z)=\chi \circ h(z)$. Inserting $F_{q, \epsilon}$ into display (3.5) in the proof of Proposition 3.1 and following the rest of the argument in that proof gives the functions claimed in the proposition. 
As in Proposition 3.2 of [Mc1, the functions in Proposition 3.1 may be summed to give a certain family of bounded plurisubharmonic functions in a strip near $b \Omega$. Theorem 2.2 in [Cat] then shows that (7) holds with $\kappa=1 / M$ on a neighborhood of each $p \in b \Omega$. To establish (7), with uniform constituents, on the domains $\Omega_{q, \epsilon}$, we use the argument in Proposition 4.1. For clarity, we amplify this argument. Temporarily fix $q$ and $\epsilon$, let $\delta>0$ be given, and let $\left(z_{1}, \ldots, z_{n}\right)$ be the $(q, \delta \epsilon)$ extremal coordinates. Let $S(=S(q, \epsilon, \delta))$ be the scaling map in these coordinates with scale factors $\tau_{1}(q, \epsilon), \ldots, \tau_{n}(q, \epsilon)$ :

$$
z=S(w)=\left(\tau_{1}(q, \epsilon) w_{1}, \ldots, \tau_{n}(q, \epsilon) w_{n}\right) .
$$

If $F=F_{q, \delta \epsilon}$ is the function given by Proposition 3.1, it follows from (vi) in Proposition 1 that

$$
\begin{aligned}
\sum_{k, l=1}^{n} \frac{\partial^{2}}{\partial w_{k} \partial \bar{w}_{l}}(F \circ S)(w) \xi_{k} \bar{\xi}_{l} & \gtrsim \sum_{j=1}^{n} \frac{\left|\tau_{j}(q, \epsilon) \xi_{j}\right|^{2}}{\tau_{j}(q, \delta \epsilon)^{2}} \\
& \gtrsim \delta^{-\frac{2}{M}}\|\xi\|^{2}, \quad \text { if } S(w) \in P_{\delta \epsilon}(q) .
\end{aligned}
$$

Furthermore, $F \circ S$ is supported in $\left\{w: S(w) \in P_{\delta \epsilon}(q)\right\}$. Note that $S^{-1}\left(P_{\delta \epsilon}(q)\right)=$ $\left\{w:\left|w_{j}\right| \leq \frac{\tau_{j}(q, \delta \epsilon)}{\tau_{j}(q, \epsilon)}\right\}$. The properties in Proposition 1 (ii) and (iii) for the polydiscs $P_{\epsilon}(q)$ also hold for the sets $S^{-1}\left(P_{\delta \epsilon}(q)\right)$; this follows by combining (i) and (iv) with (ii) and (iii) in Proposition 1.

Consider the strip near $b \Omega_{q, \epsilon}$ of thickness $\delta$ :

$$
C_{\delta}=\left\{-\delta<\rho_{\epsilon}<0\right\}
$$

It follows from the remarks above (see the proof of Proposition 3.2 in Mc1]) that $C_{\delta} \cap V$ may be covered by a finite number of sets $S^{-1}\left(P_{\delta \epsilon}\left(q_{1}\right)\right), \ldots, S^{-1}\left(P_{\delta \epsilon}\left(q_{N}\right)\right)$, where $N=N(\delta)$, but where the number of the sets $S^{-1}\left(P_{\delta \epsilon}\left(q_{j}\right)\right)$ that intersect another $S^{-1}\left(P_{\delta \epsilon}\left(q_{k}\right)\right)$ is bounded independent of $q, \epsilon$, and $\delta$. Adding up the functions $F_{q_{j}, \delta \epsilon}$ gives a plurisubharmonic function $\lambda_{\delta}$ satisfying

(a) $\left|\lambda_{\delta}\right| \lesssim 1$ on $\Omega_{q, \epsilon} \cap V$

(b) $\partial \bar{\partial} \lambda_{\delta}(z)(\xi, \bar{\xi}) \gtrsim \delta^{-\frac{2}{M}}\|\xi\|^{2}, z \in C_{\delta}$.

Theorem 2.2 in Cat then implies that (7) holds, with $\kappa=1 / M$, uniformly on the domains $\Omega_{q, \epsilon}$.

\section{REFERENCES}

[B-C-D] J. Bruna, P. Charpentier \& Y. Dupain, Zero varieties for the Nevanlinna class in convex domains of finite type in $\mathbb{C}^{n}$, Ann. of Math 147 (1998), 391-415. MR 99e:32023

[B-N-W] J. Bruna, A. Nagel \& S. Wainger, Convex hypersurfaces and Fourier transforms, Ann. of Math. 127 (1988), 333-365. MR 89d:42023

[Cat] D. Catlin, Subelliptic estimates for the $\bar{\partial}$-Neumann problem on pseudoconvex domains, Ann. of Math. 126 (1986), 131-191. MR 88i:32025

[Cum1] A. Cumenge, Sharp estimates for $\bar{\partial}$ on convex domains of finite type, Ark. Mat. 39 (2001), 1-25.

[Cum2] Zero sets of functions in the Nevanlinna class in convex domains of finite type (preprint).

[DiFo] K. Diederich \& J.E. Fornæss, Support functions for convex domains of finite type, Math. Zeit. 230 (1999), 145-164. MR 2000b:32024

[FoSi] J.E. Fornæss \& N. Sibony, Construction of P.S.H. functions on weakly pseudoconvex domains, Duke Math. J. 58 (1989), 633-655. MR 90m:32034

[KrLi] S.G. Krantz \& S.-Y. Li, Duality theorems for Hardy and Bergman spaces on convex domains of finite type, Ann. Inst. Fourier 45 (1995), 1305-1327. MR 96m:32002 
[Mc1] J. McNeal, Estimates on the Bergman kernels of convex domains, Adv. Math. 109 (1994), 108-139. MR 95k:32023

[Mc2] Convex domains of finite type, J. Funct. Anal. 108 (1992), 361-373. MR 93h:32020

[McSt] J. McNeal \& E.M. Stein, The Szegö projection on convex domains, Math. Zeit. 224 (1997), 519-553. MR 98f:32023

Department of Mathematics, Ohio State University, Columbus, Ohio, 43210

E-mail address: mcneal@math.ohio-state.edu 